\title{
Affinage et qualité du Gruyère de Comté I. Protocole expérimental. Caractérisation
des conditions d'affinage et des ateliers de fabrication
}

\author{
R. GRAPPIN, J.L. BERDAGUE *, A. DASEN et R. JEUNET \\ avec la collaboration technique de G. DUBOZ \\ I.N.R.A., Station expérimentale laitière, 39800 Poligny (France) \\ * Institut Technique du Gruyère
}

\section{Résumé}

Cet article est le premier d'une série décrivant l'évolution des principales caractéristiques physico-chimiques du Gruyère de Comté au cours de l'affinage, et étudiant l'influence des conditions d'affinage, en fonction de l'origine des fromages (fromageries) et de la saison, sur la qualité du produit.

L'étude a porté sur 96 fromages représentant 24 lots de 4 fromages issus de la même cuve de fabrication dans 4 fromageries. Chacun des quatre fromages de chaque lot a été affiné dans des conditions différentes. Le schéma expérimental constitue un plan factoriel à 3 facteurs et plusieurs niveaux : affinage (4 niveaux), fromagerie (4 niveaux) et saison (2 niveaux).

Cet article décrit le protocole expérimental et les conditions d'affinage, caractérisées par la durée et la température d'affinage, la quantité de sel apportée et la composition de l'atmosphère des caves d'affinage (humidité, $\mathrm{CO}_{2}$ et $\mathrm{NH}_{3}$ ). Il donne également les principaux résultats analytiques caractérisant les laits et les conditions de fabrication des fromages.

Aux différents stades d'affinage, les fromages ont été soumis aux analyses suivantes: $\mathrm{pH}$, extrait sec, graisse, $\mathrm{Ca}, \mathrm{NaCl}$, galactose, lactates $\mathrm{L}$ et $\mathrm{D}$, acides gras volatils, évaluation de la protéolyse par dosage des fractions azotées, HPLC et électrophorèse, tests rhéologiques, examens au microscope électronique, dosage des composés d'arômes et évaluation sensorielle.

Mots clés : Fromage - Gruyère de Comté - Affinage - Matériels - Méthodes.

Ce programme a reçu le soutien financier de la Direction des Industries Agricoles et Alimentaires du Ministère de l'Agriculture (Contrat DIAA $\mathrm{n}^{\circ}$ 81/27) et de l'Association Nationale pour la Recherche et la Technologie (Bourse CIFRE de J.L. Berdagué, convention $n^{\circ}$ 92/82). 


\section{Summary}

Ripening and quality of Gruyère of Comté cheese

I. Experimental protocol. Characterization of the ripening and cheese making conditions

This paper is the first of a series describing changes in the physico-chemical characteristics of Gruyère of Comté cheese during ripening, and assessing the influence of the ripening conditions, according to the origin of the cheese (cheese plant) and the season of making, on the final quality of the product.

A number of 96 cheeses, representing 24 batches of 4 cheeses were made during 6 months, in 4 different cheese plants. Cheeses from each batch were ripened in different conditions. The experimental protocol constitutes a factorial design with 3 factors and several levels: ripening conditions ( 4 levels), cheese plant (4 levels) and season (2 levels).

This paper describes the ripening conditions, characterized by the time and temperature of ripening, the amount of salt added, and the gas composition of the ripening room atmosphere (humidity, $\mathrm{CO}_{2}, \mathrm{NH}_{3}$ ).

The main analytical results concerning the manufacturing milk and cheese making conditions are given.

The following cheese analyses were performed at different ripening stages: $\mathrm{pH}$, total solids, fat, $\mathrm{Ca}, \mathrm{NaCl}$, galactose, $\mathrm{L}$ and $\mathrm{D}$ lactate, volatile fatty acids, proteolysis, flavour compounds and sensory evaluation.

Key words : Cheese - Gruyère of Comté - Ripening - Experimental design.

\section{Introduction}

Fromage d'appellation d'origine dont la production est limitée à la région de Franche-Comté, le Gruyère de Comté ou Comté représente une production annuelle de 37000 tonnes, soit $46 \%$ du lait transformé dans le département du Doubs et $76 \%$ dans celui du Jura. C'est un fromage à pâte pressée cuite, affiné au moins 90 jours et fabriqué à partir de lait cru livré souvent deux fois par jour par les producteurs de lait. Le Comté est produit par environ 380 coopératives fromagères. Après un séjour à la fromagerie de 2 à 4 semaines en cave froide à $10-14^{\circ} \mathrm{C}$, les fromages sont regroupés dans des caves d'affinage où ils sont affinés au moins 8 à 10 semaines en cave chaude à $16-18{ }^{\circ} \mathrm{C}$, puis stockés à basse température jusqu'au moment de leur commercialisation. Il y a environ une vingtaine d'affineurs coopératifs ou privés pour l'ensemble de la production du Comté. Actuellement, les soins en cave d'affinage consistent à traiter le Comté en surface (salage, frottage, retournements) et à moduler la durée et la température d'affinage en fonction des connaissances empiriques du comportement des fromages en cave. Ce comportement est suivi par percussion (renseignements sur l'ouverture, la lainure) ou à l'aide de sondes (renseignements sur la texture, les défauts et les qualités organoleptiques) ou visuellement (forme du fromage, gonflements...). 
Le terme "ambiance des caves » communément utilisé est symptomatique de la méconnaissance actuelle des conditions d'affinage des fromages. Si la température est la plupart du temps régulée, l'hygrométrie est en général mal maîtrisée et la composition et la variabilité des principaux constituants de l'atmosphère des caves restent pratiquement inconnues. Il en résulte que le rôle de ces différents paramètres d'affinage sur la composition et les propriétés physiques des fromages, et par voie de conséquence sur leur qualité est mal défini, rendant difficile l'identification des causes de qualité défectueuse ; qu'il s'agisse de problèmes liés à la qualité du lait, à une technologie de fabrication mal adaptée ou à un affinage inadéquat.

$\mathrm{Si}$ on dispose d'une littérature relativement abondante (revue de MocQUOT, 1979) sur les processus biochimiques de l'affinage de l'Emmental ou du Swiss-cheese, il y a en revanche très peu d'information sur l'affinage des fromages emmorgés de type Gruyère de Comté ou Beaufort.

Un des premiers objectifs de ce travail est de décrire l'évolution normale des principales caractéristiques physico-chimiques et biochimiques du fromage, depuis sa fabrication jusqu'à sa commercialisation, afin d'obtenir des valeurs de référence sur l'affinage du Comté. En dehors de cet aspect purement analytique, indispensable pour une meilleure connaissance des mécanismes d'affinage, le protocole expérimental a été établi de façon à préciser l'influence de plusieurs types d'affinage habituellement pratiqués sur la qualité du Comté. Cette influence peut varier en fonction de l'origine des fromages, c'est-à-dire de la qualité du lait et des paramètres de fabrication (facteur fromagerie), et en fonction de la saison de fabrication.

Par le biais de cette étude, il devrait également être possible de recueillir des données sur les paramètres mesurables spécifiques de l'affinage, pour définir éventuellement leur influence sur la composition et la qualité des fromages.

Enfin, le dernier objectif de ce travail a été d'essayer de relier les caractéristiques analytiques des fromages mesurées à différents stades de l'affinage, à la qualité finale du produit définie par des analyses sensorielles.

La connaissance simultanée, d'une part des relations entre variables de composition et de qualité des fromages, et d'autre part de l'influence des facteurs sur ces variables, devrait apporter une meilleure compréhension des mécanismes qui concourent à la qualité du produit affiné. Cela devrait permettre également, à partir d'un nombre limité d'analyses et de tests effectués à un stade précoce de l'affinage, de prévoir certains aspects de la qualité finale des fromages en fonction des conditions d'affinage.

En effet, alors qu'on dispose maintenant de techniques analytiques permettant de mesurer de façon rapide, précise, et souvent à un coût relativement faible, un grand nombre de paramètres de composition, il devrait être possible de conduire l'affinage autrement que d'une manière empirique, sachant qu'une maîtrise de l'affinage passe obligatoirement par une connaissance des caractéristiques physico-chimiques du fromage avant son entrée en cave chaude et par un suivi des modifications biochimiques du fromage en cours d'affinage.

Dans ce premier article, nous exposerons le plan d'expérience, le protocole de prélèvement des échantillons et la nature des analyses effectuées sur 
les fromages en cours d'affinage, et nous décrirons succinctement les méthodes statistiques utilisées. Nous indiquerons ensuite la nature et les résultats des contrôles qui ont permis de caractériser, d'une part les conditions d'affinage, et d'autre part la qualité des laits et les techniques de fabrication des fromages.

Mis à part un tableau donnant les principales caractéristiques physicochimiques des fromages à 20 heures, la description des techniques analytiques employées, ainsi que les résultats concernant le rôle des différents facteurs sur l'évolution biochimique des fromages en cours d'affinage seront données séparément dans une série d'articles qui porteront respectivement sur :

- le $\mathrm{pH}$ et les composants majeurs du fromage (extrait sec, calcium, chlorure de sodium);

- la fermentation lactique et les acides gras volatils;

- la protéolyse ;

- les produits d'arôme ;

- la qualité du fromage.

\section{Matériel et méthodes}

\section{A. Plan d'expérience}

L'objectif essentiel de l'expérimentation étant de déterminer le rôle de l'affinage sur la qualité du Comté, les quatre industriels affineurs (A1, A2, A3 et $\mathrm{A} 4$ ) qui ont participé au programme ont été choisis de façon à représenter l'éventail le plus large possible des conditions d'affinage du Comté. Les essais ont été faits dans quatre fromageries (F1, F2, F3 et F4) et chacun des 4 affineurs a reçu, lors de chaque fabrication, un fromage issu d'une cuve multiple à quatre fromages. Les fromages issus d'une même cuve sont considérés comme ayant des caractéristiques physico-chimiques identiques. Les fabrications ont été distribuées sur deux périodes de trois mois chacune, février, mars et avril pour l'hiver, et août, septembre et octobre pour l'été. Chaque mois, quatre séries de fromages ont été faites pendant quatre jours consécutifs, par chaque fromagerie. Sur les 384 fromages fabriqués, seuls 96 , représentant 1 journée de fabrication par fromagerie et par mois ont fait l'objet d'analyses chimiques et sensorielles, les autres fromages n'ont été soumis qu'à un classement technique de qualité.

L'essentiel de l'étude constitue donc un plan factoriel à 3 facteurs dont les niveaux sont répartis de la façon suivante : fromagerie (4 niveaux), affinage (4 niveaux) et saison de fabrication ( 2 niveaux), avec 3 répétitions (mois) par saison. La durée de l'affinage a été fixée par l'affineur en fonction de l'état de maturité des fromages.

\section{B. Contrôles effectués sur les fromages}

\section{Modalités d'échantillonage}

Les analyses chimiques et bactériologiques ainsi que les analyses sensorielles et les notations de qualité ont porté sur la totalité des 96 fromages de 
l'étude. Sur ce nombre, 24 fromages seulement représentant les fabrications d'une seule fromagerie (F4), ont fait l'objet d'analyses particulières : dosage des composés d'arômes, tests rhéologiques, examen au microscope électronique et dosage des protéines par chromatographie liquide haute performance.

Les prélèvements et analyses ont été faits aux stades suivants :

- Stade F (fromagerie) : 20 heures après fabrication ;

- Stade A (avant affinage) : 3 semaines en moyenne après fabrication et avant entrée en cave chaude ;

- Stade $\mathrm{P}$ (pendant affinage) : 9 semaines après fabrication ;

- Stade C (coupe): en fin d'affinage, soit entre 4 et 9 mois après fabrication, suivant le type d'affinage.

a) Localisation et calendrier des prélèvements sur les fromages

La localisation des prélèvements, schématisée dans la figure 1 , dépend du stade d'affinage auquel sont faits les prélèvements et du type d'analyse effectuée.

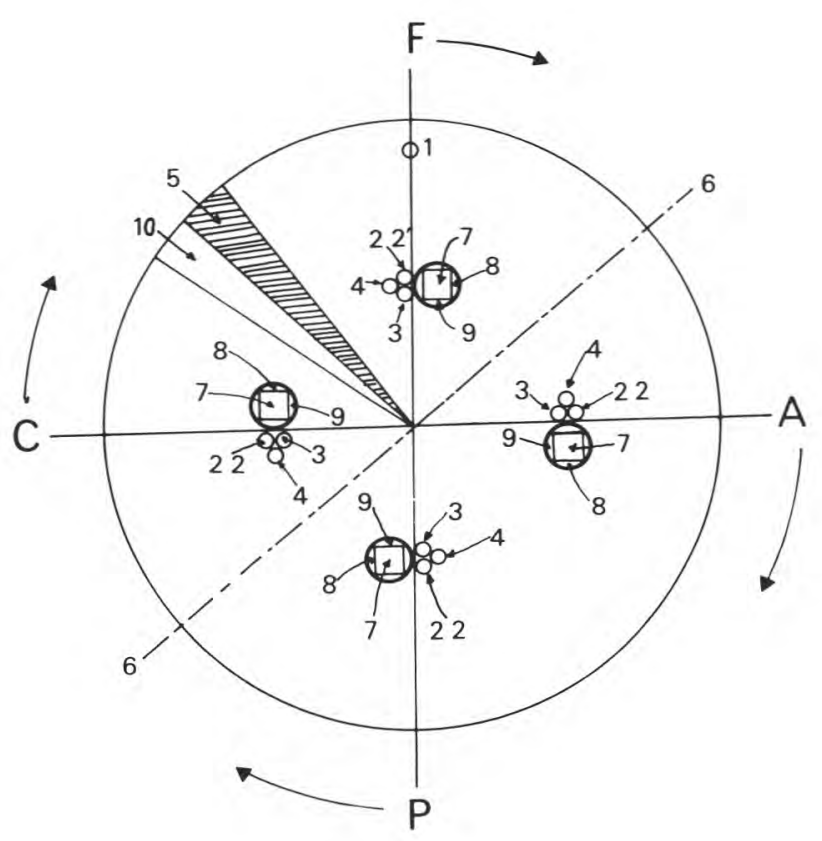

Fig. 1

Localisation sur la meule de fromage des prélèvements d'échantillons destinés aux analyses (voir texte).

Localization on the cheese wheel of samples used for analysis (see text). 
Les mesures de $\mathrm{pH}$, de galactose et d'extrait sec au stade $\mathrm{F}$ sont faites sur le mélange des sondes 1 (talon) et 2 (milieu de rayon) afin de tenir compte des hétérogénéités existantes dans le fromage à 20 heures (WILDBRETT et Kiermeier, 1960). Dans tous les autres cas, les sondes sont prélevées en milieu de rayon (sondes $2,2^{\prime}, 3,4,7,8,9$ ).

Au stade coupe, les portions 5 et 10 représentant chacune un secteur prélevé dans le quadrant $(\mathrm{C}-\mathrm{F})$ sont destinées à l'analyse sensorielle et à l'analyse des arômes. Le plan de coupe 6 servira aux notations de présentation des fromages.

\section{b) Prélèvements et conservation des échantillons}

Les prélèvements ont nécessité deux types de sondes en acier inoxydable : une sonde de diamètre interne de $20 \mathrm{~mm}$ pour les prélèvements $1,2,3$, 4 et une sonde de diamètre interne de $140 \mathrm{~mm}$ pour les prélèvements $7,8,9$.

Le prélèvement $2^{\prime}$, stérile, est obtenu à partir de la sonde 2 prélevée ellemême stérilement après raclage et désinfection locale à l'alcool des deux faces du fromage. Les prélèvements $7,8,9$ sont taillés dans le cylindre de fromage (diamètre $140 \mathrm{~mm}$ ). Le prélèvement 7 (rhéologie) forme un parallélépipède rectangle de section carrée. Les prélèvements 8 et 9 sont deux des quatre parties enlevées au cylindre de $140 \mathrm{~mm}$ pour former les prélèvements 7 .

Tous les échantillons prélevés (excepté $2^{\prime}, 5$ et 10 ) sont emballés dans du papier aluminium sulfurisé, l'aluminium étant du côté du fromage afin de limiter les transferts de soluté lors de la congélation. L'échantillon $2^{\prime}$ est placé dans un tube en plastique stérile. Les échantillons destinés à l'analyse sensorielle et aux arômes ( 5 et 10$)$ sont emballés dans le même type de papier, le côté sulfurisé étant placé contre le fromage pour éviter les risques de goût de métal et d'oxydation.

Après prélèvement en cave de fromagerie ou en cave d'affinage, les échantillons sont placés à $4{ }^{\circ} \mathrm{C}$ jusqu'au moment de l'analyse pour la mesure du $\mathrm{pH}$ et pour les analyses chimiques (extrait sec, fractions azotées, calcium, chlorure de sodium) ou microbiologiques. Les échantillons sont congelés à $-25^{\circ} \mathrm{C}$ pour les dosages de sucres, lactates, acides gras volatils, pour l'analyse par électrophorèse, HPLC et l'analyse des composés volatils d'arôme.

L'analyse en microscopie électronique et les tests rhéologiques ont été effectués 24 heures après prélèvement, après transport en caisse isotherme à une température variant entre 8 et $12^{\circ} \mathrm{C}$.

Après découpe selon le plan 6 , les fromages sont photographiés et les portions 5 destinées à l'analyse sensorielle sont conservées au maximum $24 \mathrm{~h}$ en chambre froide $\left(4^{\circ} \mathrm{C}\right)$ jusqu'au moment de la dégustation.

\section{Analyses}

Le tableau 1 résume la nature des analyses qui ont été faites sur les fromages aux différents stades d'affinage. Sans compter les tests rhéologiques, les analyses par HPLC, les examens microscopiques et le dosage des composés d'arômes, ce sont 22 variables physico-chimiques qui serviront à caractériser les fromages au cours de leur affinage. 
TABLEAU I

Localisation des prélèvements (voir figure 1), nature et répartition en cours d'affinage des analyses faites sur fromage

Localization of sampling (see figure 1), nature and distribution throughout ripening of cheese analyses

\begin{tabular}{|c|c|c|}
\hline $\begin{array}{c}\text { Identification } \\
\text { du prélèvement } \\
\text { Identification } \\
\text { Number of sampling } \\
\end{array}$ & $\begin{array}{l}\text { Stade d'affinage } \\
\text { Stage of ripening }\end{array}$ & $\begin{array}{l}\text { Nature des analyses } \\
\text { Nature of analyses }\end{array}$ \\
\hline 1,2 & $\begin{array}{l}\mathrm{F} \\
\mathrm{A}, \mathrm{P}, \mathrm{C}\end{array}$ & $\begin{array}{l}\mathrm{pH}, \text { Extrait sec } \\
\mathrm{pH}, \text { total solids }\end{array}$ \\
\hline 1,2 & F & $\begin{array}{l}\text { Matière grasse } \\
\text { Fat }\end{array}$ \\
\hline 2 & $\mathrm{~F}, \mathrm{~A}, \mathrm{P}, \mathrm{C}$ & $\begin{array}{l}\text { Calcium, Chlorure de sodium } \\
\text { Calcium, sodium chloride }\end{array}$ \\
\hline 1,2 & $\begin{array}{l}\text { F } \\
\text { A }\end{array}$ & $\begin{array}{l}\text { Galactose } \\
\text { Galactose }\end{array}$ \\
\hline 2 & $\mathrm{~F}, \mathrm{~A}, \mathrm{P}, \mathrm{C}$ & $\begin{array}{l}\text { Lactate } \mathrm{L} \text { et } \mathrm{D} \\
L \text { and } D \text { Lactate }\end{array}$ \\
\hline 4 & $\mathrm{~A}, \mathrm{P}, \mathrm{C}$ & $\begin{array}{l}\text { Acides gras volatils } \\
\text { Volatile fatty acids }\end{array}$ \\
\hline 2 & $\mathrm{~F}, \mathrm{~A}, \mathrm{P}, \mathrm{C}$ & $\begin{array}{l}\text { Azote total et fractions azotées } \\
\text { Total nitrogen and nitrogen fractions }\end{array}$ \\
\hline 2,3 & $\mathrm{~F}, \mathrm{~A}, \mathrm{P}, \mathrm{C}$ & $\begin{array}{l}\text { Electrophorèse } \\
\text { Electrophoresis }\end{array}$ \\
\hline $2^{\prime}$ & $\mathrm{F}, \mathrm{A}, \mathrm{P}, \mathrm{C}$ & $\begin{array}{l}\text { Microbiologie } \\
\text { Microbiology }\end{array}$ \\
\hline 5 & $\mathrm{C}$ & $\begin{array}{l}\text { Analyse sensorielle } \\
\text { Sensory analysis }\end{array}$ \\
\hline 6 & $\mathrm{C}$ & $\begin{array}{l}\text { Notation de présentation } \\
\text { Grading }\end{array}$ \\
\hline 7 & $\mathrm{~F}, \mathrm{~A}, \mathrm{P}, \mathrm{C}$ & $\begin{array}{l}\text { Test rhéologique } \\
\text { Rheological test }\end{array}$ \\
\hline 8 & $\mathrm{~F}, \mathrm{~A}, \mathrm{P}, \mathrm{C}$ & $\begin{array}{l}\text { Dosage des protéines par HPLC } \\
\text { Protein test by HPLC }\end{array}$ \\
\hline 9 & $\mathrm{~F}, \mathrm{~A}, \mathrm{P}, \mathrm{C}$ & $\begin{array}{l}\text { Microscopie électronique } \\
\text { Electron microscopy }\end{array}$ \\
\hline 10 & $\mathrm{C}$ & $\begin{array}{l}\text { Dosage composés d'arômes } \\
\text { Analysis flavour compounds }\end{array}$ \\
\hline
\end{tabular}




\section{Analyses statistiques}

\section{Statistiques élémentaires}

Pour chaque variable sont présentées les moyennes et les écart-types calculés aux différents stades d'affinage.

\section{Relations entre variables}

Une matrice des corrélations simples a été calculée pour chaque stade sur l'ensemble des données analytiques. Seuls les coefficients dont le seuil de signification est supérieur à $1 \%$ pour les variables physico-chimiques et $5 \%$ pour les variables sensorielles ont été retenus.

Lorsque des relations permettent d'obtenir une vision synthétique d'un mécanisme, deux types de présentation des résultats ont été retenus :

- La régression linéaire, simple ou multiple, qui donne la relation entre une ou plusieurs variables explicatives $\left(\mathrm{x}_{\mathrm{i}}\right)$ et une variable à expliquer $(\mathrm{y})$.

Cette relation est de la forme : $y=a_{i} x_{i}+a_{2} x_{2}+\ldots a_{n} x_{n}+a_{0}$ où $a_{i}$ est le coefficient de régression et $\mathrm{a}_{0}$ le terme constant.

- L'analyse en composantes principales qui est une technique statistique multidimensionnelle, dont le but premier est de condenser l'essentiel des informations fournies par une série de $n$ variables interdépendantes (ou variables initiales), en un nombre restreint de variables indépendantes ou composantes principales (axes), représentées par un espace à une, deux ou trois dimensions. La signification de ces axes, qui sont des combinaisons linéaires des variables initiales, est faite sur le cercle des corrélations par l'étude des corrélations existantes entre ces axes et les variables initiales. La projection des individus dans l'espace ainsi défini permet d'étudier la forme du nuage de points et de vérifier la signification attribuée à chaque axe.

Lorsque des variables interviennent directement dans le calcul des axes d'une analyse factorielle, on dit qu'il s'agit de variables actives. Le comportement d'une ou plusieurs autres variables vis-à-vis des variables actives peut être établi en représentant ces variables en fonction de leurs corrélations avec les axes sans les faire intervenir dans les calculs qui définissent ces axes. On parle alors de variables supplémentaires.

\section{L'étude des facteurs}

L'influence respective des différents facteurs sur la composition et sur la qualité finale des fromages a été estimée grâce à l'analyse de variance, et visualisée par l'analyse en composantes principales et l'analyse factorielle des correspondances simples.

Analyses de variance : nous avons considéré que les niveaux des facteurs étudiés correspondaient à un modèle fixe. Toutes les combinaisons des différents facteurs ayant le même nombre de répétitions, nous avons à faire à un modèle d'analyse de variance croisée à dispositif en blocs équilibrés orthogonaux. 
Les analyses sont réalisées suivant le modèle mathématique :

$\mathrm{Y}_{\mathrm{i}, \mathrm{j}, \mathrm{k}, \mathrm{I}}=\mu+\alpha_{\mathrm{i}}^{\mathrm{A}}+\beta_{\mathrm{j}}^{\mathrm{F}}+\gamma_{\mathrm{k}}^{\mathrm{S}}+(\alpha \beta)_{i, j}^{\mathrm{AF}}+(\alpha \beta)_{i, \mathrm{k}}^{\mathrm{AS}}+(\beta \gamma)_{j, k}^{\mathrm{FS}}+\mathrm{R}_{\mathrm{i}, \mathrm{j}, \mathrm{k}, \mathrm{I}}$

$\mathrm{Y}_{\mathrm{i}, \mathrm{j}, \mathrm{k}, \mathrm{l}}$ : valeur de la caractéristique du fromage

$\mu \quad$ : terme constant à tous les fromages

$\alpha_{\mathrm{i}}^{\mathrm{A}}$ : effet moyen du facteur affinage

$\beta_{j}^{F} \quad$ : effet moyen du facteur fromagerie

$\gamma_{\mathrm{k}}^{\mathrm{s}}$ : effet moyen du facteur saison

$(\alpha \beta)_{i, j}^{A F},(\alpha \gamma)_{i, k}^{\mathrm{AS}},(\beta \gamma)_{i, k}^{\mathrm{FS}}$ : terme moyen des interactions

1: nombre de répétitions

$\mathrm{R}_{\mathrm{i}, \mathrm{k}, \mathrm{l}}$ : terme aléatoire qui permet non seulement de juger l'effet des facteurs mais aussi celui des interactions entre facteurs.

L'influence respective de chaque facteur, outre leur signification statistique pour le test $\mathrm{F}$, est estimée en examinant le pourcentage de la variance expliqué par chaque facteur et chaque interaction.

Analyse en composantes principales : cette méthode déjà mentionnée permet, en indiçant judicieusement les individus en fonction du facteur à étudier, de visualiser au niveau individuel, l'influence des facteurs sur les variables de composition ou de qualité des fromages utilisées dans l'analyse.

Analyse factorielle des correspondances simples: il s'agit essentiellement d'un mode de présentation graphique de tableaux de contingence. Elle vise à rassembler en un ou plusieurs graphes la plus grande partie possible de l'information contenue dans un tableau de contingence, en s'attachant, non pas aux valeurs absolues, mais aux correspondances entre les caractères, c'est-àdire aux valeurs relatives.

Les analyses statistiques ont été réalisées d'une part à l'aide de la programmathèque AMANCE (BACHACOU et al., 1981) adaptée au microordinateur (Mini 6-CII) et d'autre part, sur micro-ordinateur MICRAL 80-21 C à partir des logiciels MICROSTAT, élaborés par ITCF (1984).

\section{Caractérisation des fromageries et de la saison de fabrication}

\section{Situation géographique et équipement des fromageries}

- Altitude : les fromageries F1 et F2 sont situées sur le premier plateau jurassien entre 400 et $600 \mathrm{~m}$ d'altitude, F3 et F4 sont des fromageries de montagne localisées entre 850 et $1100 \mathrm{~m}$ d'altitude.

- Collecte du lait : deux fromageries, F2 et F4, travaillent en report traditionnel de 12 heures du lait de la traite du soir, les autres fromageries, F1 et F3, opèrent avec des quantités variables de lait de report de 24 heures.

- Equipement matériel des fromageries: les quatre fromageries sont équipées de cuves multiples (4 fromages minimum); F1 et $\mathrm{F} 2$ possèdent un équipement de soutirage sous vide, tandis que F3 et F4 soutirent par pompe à caillé. 
- Levains : les fromageries F1, F2, F3 utilisent plusieurs " cultures sauvages » préparées à partir de lactosérum cru ou à partir de lactosérum désalbuminé ensemencé avec le levain de la veille. Ces sérums sont incubés soit à $30^{\circ} \mathrm{C}, 5-18 \mathrm{~h}$, pour favoriser la croissance des streptocoques, soit à $42{ }^{\circ} \mathrm{C}, 8-18 \mathrm{~h}$, pour favoriser celle des lactobacilles. La fromagerie $\mathrm{F} 4$ utilise uniquement des ferments sélectionnés cultivés sur lactosérum enrichi avec $25 \%$ de lait et traité thermiquement $75{ }^{\circ} \mathrm{C}, 10 \mathrm{mn}$, pour $S$. thermophilus et sur lactosérum désalbuminé $\left(98^{\circ} \mathrm{C}-20 \mathrm{mn}\right)$ pour $L$. helveticus. L'enzyme coagulante est introduite sous forme de caillette de veau dans le lactosérum cru ou désalbuminé.

Pendant toute l'expérimentation, F1, F2 et F3 ont pratiqué une maturation courte du lait de fabrication par apport d'un levain lactique mésophile préparé à partir de lactosérum cru, tandis que F4 n'a utilisé un levain lactique mésophile sélectionné que pendant l'hiver. Dans ce dernier cas, la maturation s'effectuait uniquement sur la traite du soir au cours de sa conservation.

\section{Prélèvement et analyse des laits et des levains}

a) Lait: les prélèvements sont effectués, soit avant addition de présure pour les analyses chimiques, soit après emprésurage pour tenir compte des apports microbiologiques liés à l'addition de présure, dans le cas des analyses microbiologiques ;

- azote et fractions azotées: les fractions azote soluble (NS) et azote non protéique (NNP) ont été préparées selon la technique de Rowland (1938). L'azote est dosé par la méthode Kjeldahl (Projet de Norme FIL E-Doc 214, 1985 ) et les résultats, donnés en $\mathrm{g} / \mathrm{kg}$, sont exprimés de la façon suivante :

- taux de matière azotée totale (T.MAT) $=\mathrm{N}$ total $\times 6,38$,

- taux de protéines $(\mathrm{TP})=\mathrm{N}$ (total - NNP) 6,38,

- taux de caséines (T.Cas) $=(\mathrm{N}$ total $-\mathrm{NS}) 6,38$,

- taux matière azotée non protéique $(\mathrm{ANP})=\mathrm{NNP} \times 6,38$;

— matière grasse : méthode Gerber (Norme NF V-04 210); (1977) ;

- calcium: dosage complexométrique selon la technique de Pearce

- plasmine et plasminogène : dosage selon la technique de RichardSON et Pearce (1981). Les résultats sont exprimés en unité de substrat AMC consommés par ml de lait ( 1 unité $\mathrm{AMC}=10^{-9}$ Mole d'Amino-Méthyl-Coumarine par $\mathrm{mn}$ et par $\mathrm{ml}$ de lait);

- analyses microbiologiques: compte tenu de la présence d'une flore lactique importante due à l'apport des levains, seules les numérations de Pseudomonas et de Coliformes ont été retenues pour caractériser la qualité bactériologique des laits de fabrication.

Pseudomonas: numération de la flore lactose négative après ensemencement en surface sur milieu Violet Red Bile Agar (VRB Difco) et incubation $24 \mathrm{~h}$ à $30^{\circ} \mathrm{C}$.

Coliformes : numération de la flore après ensemencement sur milieu VRB et incubation $18-24 \mathrm{~h}$ à $30^{\circ} \mathrm{C}$. 
b) Levains : un échantillon moyen a été reconstitué en laboratoire, à partir des proportions volumétriques des différents levains apportés dans les laits de fabrication.

- Streptocoques lactiques mésophiles et thermophiles : numération par ensemencement sur milieu M17 (DIFCO) et incubation 3 jours à $30^{\circ} \mathrm{C}$.

- Lactobacilles : numération de la flore par ensemencement en surface sur milieu MRS (DIFCO) acidifié à pH 5,4. Les boîtes sont placées dans une jarre pour culture anaérobie et incubées 3 jours à $+37^{\circ} \mathrm{C}$.

A partir de ces dénombrements et des quantités de levains apportées, nous avons calculé les quantités de bactéries lactiques (Strepto + Lacto) apportées par millilitre de lait de chaudière ainsi que la proportion de Streptocoques (\% Strepto/Lacto) par rapport à la totalité Streptocoques + Lactobacilles.

- Activité acidifiante : ensemencement à $1 \%(\mathrm{P} / \mathrm{P})$ d'un lait écrémé en poudre reconstitué à $10 \%(\mathrm{P} / \mathrm{P})$, pasteurisé $80^{\circ} \mathrm{C}, 30 \mathrm{~min}$ et incubation $5 \mathrm{~h}$ à $42{ }^{\circ} \mathrm{C}$. L'activité acidifiante des levains, exprimée en degrés Dornic $\left({ }^{\circ} \mathrm{D}\right)$, est donnée par le volume de soude $(0,1 \mathrm{~N})$ nécessaire pour neutraliser l'acidité développée dans 9 grammes de culture.

\section{Caractéristiques physico-chimiques des fromages à 20 heures}

La composition des fromages à la sortie de presse étant directement liée à celle des laits et aux techniques de fabrication, nous résumons dans le tableau 2 les principales caractéristiques physico-chimiques des fromages obtenus par chaque fromagerie et pour chaque saison.

\section{Résultats}

Les valeurs moyennes obtenues par fromagerie et par saison, pour l'ensemble des variables mesurées sur laits de fabrication et sur levains, sont résumées dans le tableau 3. Hormis un TP et un rapport T.Cas/TP légèrement plus faible pour $\mathrm{F} 1$ et un rapport MG/T.Cas plus faible pour F3 et F4, il existe peu de différence au niveau de la composition chimique moyenne des laits des quatre fromageries. Il en est de même pour la teneur en plasmine. En revanche, les fromages de F4 présentent en moyenne un gras/sec plus faible que celui des autres fromageries (tabl. 2).

Bien que F1 et F3 aient utilisé du lait de report réfrigéré $24 \mathrm{~h}$, on ne constate pas de différence très sensible par rapport aux deux autres fromageries dans les teneurs en Pseudomonas.

S'il existe une certaine homogénéité de composition des laits de fromagerie, en revanche, les différences entre saisons sont très nettes pour beaucoup de variables. Par rapport au lait d'hiver, le lait d'été est plus riche en caséine $(+1,92 \mathrm{~g} / \mathrm{kg})$, en ANP $(+0,69 \mathrm{~g} / \mathrm{kg})$ et en plasmine $(+0,14 \mathrm{AMC} / \mathrm{ml})$. Il se caractérise également par une proportion ANP/MAT plus élevée $(7,00 \%$ contre $5,52 \%$ ) et par une pauvreté relative du lait en calcium par rapport au taux de caséines. Les fromagers effectuant un taux d'écrémage relativement constant en cours d'année, on note un rapport MG/T.Cas plus faible en été, en raison d'une élévation du taux de caséines; il s'en suit une diminution du gras/sec des fromages pendant les mois d'été. La population des laits en Pseudomonas est plus importante pendant les mois d'été. 
TABLEAU II

Composition physico-chimique (moyenne et écart-type) des fromages de $20 \mathrm{~h}$ en fonction de la fromagerie et de la saison Physico-chemical composition (mean and standard deviation) of $20 \mathrm{~h}$ cheeses according to the cheese plant and the season

\begin{tabular}{|c|c|c|c|c|c|c|}
\hline \multirow{2}{*}{$\begin{array}{l}\text { Variables } \\
\text { Variables }\end{array}$} & \multicolumn{4}{|c|}{$\begin{array}{l}\text { Fromagerie } \\
\text { Cheese plant }\end{array}$} & \multicolumn{2}{|c|}{$\begin{array}{l}\text { Saison } \\
\text { Season }\end{array}$} \\
\hline & $\mathrm{F} 1$ & $\mathrm{~F} 2$ & F3 & $\mathrm{F} 4$ & $\begin{array}{l}\text { Hiver } \\
\text { Winter }\end{array}$ & $\begin{array}{c}\text { Eté } \\
\text { Summer }\end{array}$ \\
\hline $\mathrm{pH}$ & $5,31 \pm 0,04$ & $5,29 \pm 0,04$ & $5,29 \pm 0,02$ & $5,32 \pm 0,06$ & $5,28 \pm 0,05$ & $5,32 \pm 0,02$ \\
\hline $\begin{array}{l}\text { Extrait sec (3) } \\
\text { Dry matter }\end{array}$ & $62,69 \pm 0,33$ & $62,23 \pm 0,13$ & $62,74 \pm 0,64$ & $62,33 \pm 0,27$ & $62,68 \pm 0,43$ & $62,32 \pm 0,38$ \\
\hline $\begin{array}{l}\text { Gras/sec }{ }^{(1)} \\
\text { Fat in dry matter }\end{array}$ & $49,8 \pm 1,1$ & $49,5 \pm 1,11$ & $49,6 \pm 0,8$ & $48,8 \pm 0,7$ & $50,2 \pm 0,7$ & $48,7 \pm 0,6$ \\
\hline $\begin{array}{l}\text { Calcium } \\
\text { Calcium }\end{array}$ & $1,530 \pm 0,028$ & $1,515 \pm 0,017$ & $1,532 \pm 0,024$ & $1,565 \pm 0,026$ & $1,543 \pm 0,033$ & $1,528 \pm 0,025$ \\
\hline $\begin{array}{l}\text { Lactate } \mathrm{L}^{(2)} \\
\text { L lactate }\end{array}$ & $104,2 \pm 7,2$ & $105,5 \pm 9,9$ & $104,0 \pm 22,4$ & $113,3 \pm 10,9$ & $103,5 \pm 15,8$ & $110,0 \pm 12,0$ \\
\hline $\begin{array}{l}\text { Lactate } \mathrm{D}^{(2)} \\
\text { D lactate }\end{array}$ & $34,8 \pm 8,6$ & $45,7 \pm 6,3$ & $39,8 \pm 18,7$ & $17,7 \pm 3,8$ & $35,7 \pm 17,2$ & $33,2 \pm 12,6$ \\
\hline $\begin{array}{l}\text { Azote }^{(3)} \\
\text { Nitrogen }\end{array}$ & $6,89 \pm 0,14$ & $6,90 \pm 0,15$ & $6,89 \pm 0,13$ & $7,01 \pm 0,11$ & $6,81 \pm 0,08$ & $7,04 \pm 0,09$ \\
\hline $\begin{array}{l}\text { Azote soluble eau }{ }^{(3)} \\
\text { Water soluble nitrogen }\end{array}$ & $0,255 \pm 0,039$ & $0,235 \pm 0,030$ & $0,235 \pm 0,036$ & $0,200 \pm 0,048$ & $0,196 \pm 0,031$ & $0,267 \pm 0,02$ \\
\hline $\begin{array}{l}\text { NPT }^{(3)(*)} \\
P T A\end{array}$ & $0,055 \pm 0,013$ & $0,059 \pm 0,007$ & $0,060 \pm 0,082$ & $0,047 \pm 0,011$ & $0,051 \pm 0,013$ & $0,059 \pm 0,008$ \\
\hline
\end{tabular}


TABleaU III

Caractérisation physico-chimique et microbiologique des laits et des levains mis en ouvre dans les fromageries

Moyenne des résultats obtenus au cours de l'expérimentation

(6 fabrications par fromagerie et 12 fabrications par saison)

Physico-chemical and microbiological characterization of processing milks and lactic starters used in the cheese plants. Mean results of tests carried out during the experiment (average of 6 repetitions for each cheese plant and 12 repetitions for each season)

\begin{tabular}{|c|c|c|c|c|c|c|c|}
\hline \multirow{2}{*}{\multicolumn{2}{|c|}{ Variable }} & \multicolumn{4}{|c|}{$\begin{array}{l}\text { Fromagerie } \\
\text { Cheese plant }\end{array}$} & \multicolumn{2}{|c|}{$\begin{array}{l}\text { Saison } \\
\text { Season }\end{array}$} \\
\hline & & F1 & F2 & F3 & F4 & $\begin{array}{l}\text { Hiver } \\
\text { Winter }\end{array}$ & $\begin{array}{c}\text { Eté } \\
\text { Summer }\end{array}$ \\
\hline \multirow{13}{*}{ 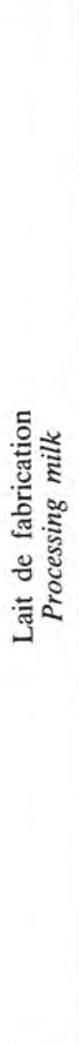 } & $\begin{array}{l}\text { Taux de protéines }(\mathrm{g} / \mathrm{kg}) \\
\text { True protein content }(\mathrm{g} / \mathrm{kg})\end{array}$ & 30,66 & 31,29 & 31,47 & 31,23 & 29,96 & 32,39 \\
\hline & $\begin{array}{l}\text { Taux de caséines }(\mathrm{g} / \mathrm{kg}) \\
\text { Casein content }(\mathrm{g} / \mathrm{kg})\end{array}$ & 25,15 & 26,13 & 26,17 & 25,94 & 24,89 & 26,81 \\
\hline & $\begin{array}{l}\text { Caséines/taux protéines }(\%) \\
\text { Casein/true protein }(\%)\end{array}$ & 82,0 & 83,5 & 83,2 & 83,1 & 83,1 & 82,7 \\
\hline & $\begin{array}{l}\text { ANP }(\mathrm{g} / \mathrm{kg}) \\
N P N(\mathrm{~g} / \mathrm{kg})\end{array}$ & 2,09 & 2,22 & 2,05 & 2,03 & 1,75 & 2,44 \\
\hline & $\begin{array}{l}\text { ANP/MAT }(\%) \\
N P N / \text { Total } N(\%)\end{array}$ & 6,38 & 6,62 & 6,12 & 6,10 & 5,52 & 7,00 \\
\hline & $\begin{array}{l}\text { Calcium }(\mathrm{g} / \mathrm{kg}) \\
\text { Calcium }(\mathrm{g} / \mathrm{kg})\end{array}$ & 1,17 & 1,21 & 1,21 & 1,21 & 1,19 & 1,21 \\
\hline & $\begin{array}{l}\text { Calcium/caséines }(\%) \\
\text { Calcium casein }(\%)\end{array}$ & 4,70 & 4,60 & 4,73 & 4,70 & 4,78 & 4,51 \\
\hline & $\begin{array}{l}\text { Taux graisse }(\mathrm{g} / \mathrm{kg}) \\
\text { Fat content }(\mathrm{g} / \mathrm{kg})\end{array}$ & 33,33 & 34,08 & 31,83 & 32,40 & 32,77 & 33,05 \\
\hline & $\begin{array}{l}\text { Graisse/caséine } \\
\text { Fat/casein }\end{array}$ & 1,33 & 1,31 & 1,22 & 1,25 & 1,32 & 1,23 \\
\hline & $\begin{array}{l}\text { Activité plasmine }(\mathrm{AMC} / \mathrm{ml}) \\
\text { Plasmin activity }(A M C / \mathrm{ml})\end{array}$ & 0,28 & 0,28 & 0,24 & 0,27 & 0,19 & 0,34 \\
\hline & $\begin{array}{l}\text { Nombre Pseudomonas }(\mathrm{UFC}) \\
\mathrm{ml}) \times 10^{3} \\
\text { Pseudomonas count }(\mathrm{CFU} / \\
\mathrm{ml}) \times 10^{3}\end{array}$ & 0,2 & 0,9 & 2,1 & 0,3 & 0,1 & 2,6 \\
\hline & $\begin{array}{l}\text { Nombre de coliformes (UFCl } \\
\mathrm{ml}) \times 10^{3} \\
\text { Coliform count }(\mathrm{CFU} / \mathrm{ml}) \times 10^{3}\end{array}$ & 11 & 33 & 48 & 9 & 25 & 33 \\
\hline & $\begin{array}{l}\text { Strepto + Lacto }(\mathrm{UFL} / \mathrm{ml}) \times 10^{6} \\
(\mathrm{CFU} / \mathrm{ml}) \times 10^{6}\end{array}$ & 0,8 & 1,2 & 0,7 & 1,2 & 0,9 & 1,0 \\
\hline \multirow{4}{*}{ 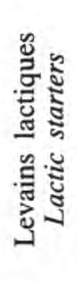 } & $\begin{array}{l}\text { Streptocoques }(\mathrm{UFC} / \mathrm{ml}) \times 10^{6} \\
\text { Streptococci }(\mathrm{CFU} / \mathrm{ml}) \times 10^{6}\end{array}$ & 220 & 263 & 166 & 155 & 177 & 189 \\
\hline & $\begin{array}{l}\text { Lactobacilles }(\mathrm{UFC} / \mathrm{ml}) \times 10^{6} \\
\text { Lactobacilli }(\mathrm{CFU} / \mathrm{ml}) \times 10^{6}\end{array}$ & 48 & 195 & 201 & 139 & 153 & 143 \\
\hline & $\begin{array}{l}\text { Proportion Strepto/Lacto (\%) } \\
\text { Ratio Strepto/Lacto (\%) }\end{array}$ & 83 & 61 & 48 & 53 & 54 & 57 \\
\hline & $\begin{array}{l}\text { Activité acidifiante }\left({ }^{\circ} \mathrm{D}\right) \\
\text { Lactic acid activity }\left({ }^{\circ} \mathrm{D}\right)\end{array}$ & 49,6 & 57,1 & 52,0 & 41,0 & 54,0 & 45,8 \\
\hline
\end{tabular}


Au niveau de la qualité des levains utilisés, F1 se distingue des autres fromageries par des levains ayant un rapport Strepto/Lacto élevé ( $83 \%$ ), et la fromagerie F4 par des levains dont l'activité acidifiante est particulièrement basse $\left(41,0^{\circ} \mathrm{D}\right.$ pour $\mathrm{F} 4$ contre $57,1^{\circ} \mathrm{D}$ pour $\mathrm{F} 2$ par exemple). Du fait des doses d'ensemencement différentes employées par les fromageries, ces fluctuations dans la qualité des levains n'entraînent pas de différences nettes au niveau des $\mathrm{pH}$ des fromages à $20 \mathrm{~h}$. Seule $\mathrm{F} 4$ se distingue par une teneur moyenne en lactate D faible (tabl. 2). Malgré des teneurs en bactries lactiques

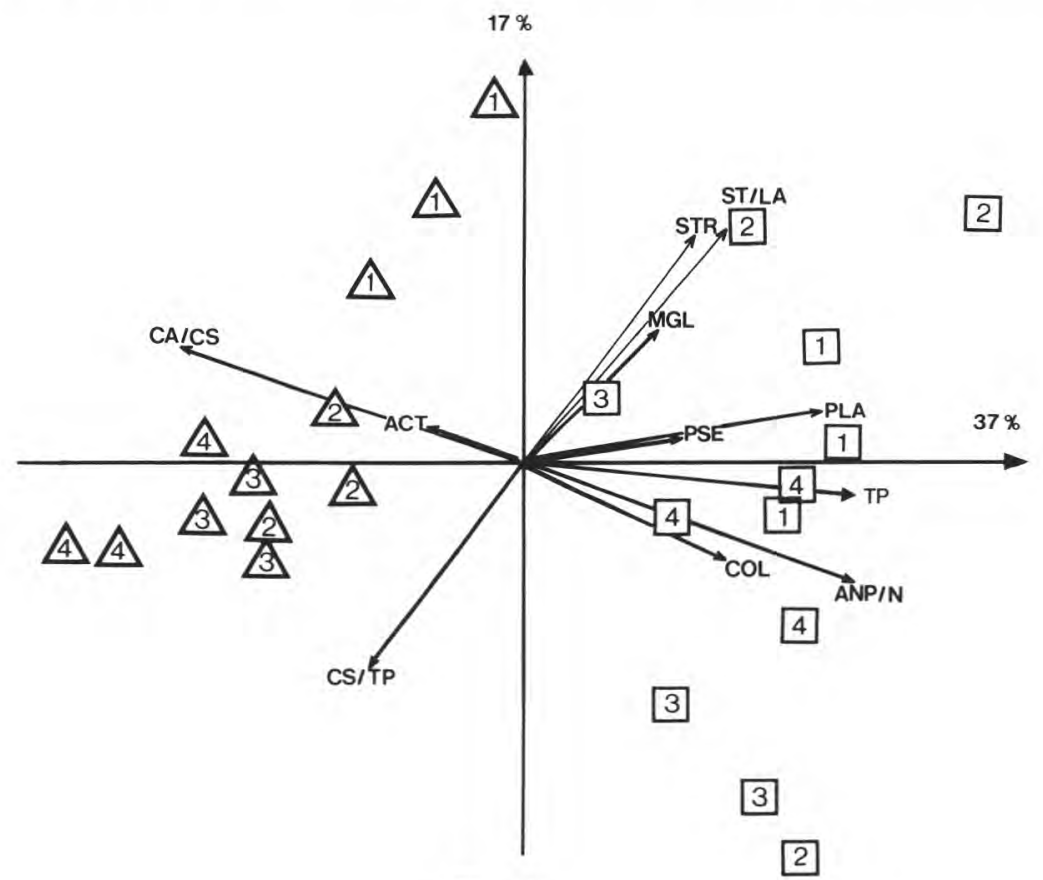

Fig. 2

Modification de la composition des laits et des levains de fromagerie, en fonction de la saison ( $\square$ : été, $\triangle$ : hiver) et de la fromagerie $(1,2,3,4)$.

Change in composition of the milk and lactic starters, according to the season $(\square:$ summer, $\triangle:$ winter $)$ and the cheese plant $(1,2,3,4)$.

Lait

Milk

$T P \quad:$ taux de protéines (true protein content)

CS/TP : rapport caséines/taux de protéines (ratio casein/true protein)

ANP/N : rapport ANP/MAT (ratio NPN/total N)

CA/CS : rapport calcium/caséine (ratio calcium/casein)

$M G L$ : taux de matière grasse (fat content)

PLA : activité de la plasmine (plasmin activity)

COL : Log nombre de coliformes (Log coliform count)

PSE : Log nombre de pseudomonas (Log Pseudomonas count)

Levains lactiques ACT: activité acidifiante des levains (Lactic acid activity of the starters)

Lactic starters STR : Log Streptocoques lactiques (Log lactic Streptococci count)

$S T / L A$ : rapport Streptocoques/Lactobacilles (ratio Streptococci/Lactobacilli) 
et des rapports Strepto/Lacto voisins, on constate que l'activité acidifiante des levains utilisés en été est plus faible que celle des levains utilisés en hiver (tabl. 2), cette activité plus faible se traduisant par des $\mathrm{pH}$ des fromages à 20 heures en moyenne légèrement plus élevés en été. Avec une moyenne de $970 \times 10^{3}$ bactéries par ml de lait (Streptocoques + Lactobacilles), on constate (tabl. 3) que les quantités de bactéries thermophiles apportées varient nettement entre fromageries mais qu'elles sont voisines entre saisons, avec toutefois sur ce dernier aspect des différences entre fromageries, puisque F2 et F3 apportent légèrement plus de levains en été qu'en hiver tandis que F1 et F4 apportent environ deux fois plus de levains en hiver qu'en été.

L'analyse en composantes principales des différentes variables qui caractérisent les fromageries et les saisons (fig. 2) permet de visualiser les informations contenues dans le tableau 3. L'axe horizontal est caractéristique de la composition chimique et l'axe vertical caractéristique des levains. L'analyse confirme la nette différence entre les laits d'été et les laits d'hiver, l'axe 2 du plan séparant les deux saisons. Par ailleurs, on constate que les fromageries sont mieux caractérisées et individualisées pendant la période hivernale que pendant l'été.

\section{E. Caractérisation du facteur affinage}

Outre la durée, les conditions d'affinage sont caractérisées essentiellement par trois paramètres mesurables : la température ou le cycle de température, la composition de l'atmosphère des caves d'affinage (humidité relative, teneur en gaz carbonique et ammoniac) et l'intensité du salage. La fréquence et la nature des soins qui peuvent influencer la nature de la morge et par voie de conséquence la qualité du fromage, n'ont pas été prises en compte, car ces paramètres sont difficilement quantifiables.

\section{Fréquences et conditions des mesures}

Les paramètres température, teneurs en gaz carbonique et en ammoniac ont été mesurés en continu 7 jours par mois dans chacune des quatre caves d'affinage. Les mesures d'humidité relative ont été effectuées de façon discontinue, à raison de 4 relevés mensuels. Les sondes de température et d'analyse des gaz ont toujours été placées au milieu du lot de fromages étudiés, situé lui-même au centre des caves afin de recevoir un traitement homogène.

a) Mesures physiques

La température a été mesurée à $\pm 0,1{ }^{\circ} \mathrm{C}$ par une sonde à thermocouple, reliée à un enregistreur MAIHAC étalonné régulièrement avec un thermomètre de précision à mercure.

L'humidité relative des caves a été suivie avec un psychromètre AHLBORN MESS. U. REGELUNGSTECHNIK, THERM 2246.

\section{b) Mesures chimiques}

Les teneurs en gaz carbonique et ammoniac ont été mesurées à l'aide d'un analyseur de gaz ULNOR-MAIHAC par photométrie infra-rouge non dispersif. Les échelles de mesure utilisées permettent des dosages dans des atmosphères contenant entre 0 et $5 \%$ de gaz carbonique (avec une précision de 
$\pm 1 \%$ de l'étendue de l'échelle de mesure), et entre 0 et $300 \mathrm{ppm}$ d'ammoniac (avec une précision de $\pm 1 \%$ de l'étendue de l'échelle de mesure).

Il est possible que les teneurs en ammoniac aient été sous-estimées systématiquement par dissolution du gaz dans la vapeur d'eau et par formation de carbonate d'ammonium.

Les teneurs en chlorure de sodium ( $\mathrm{g} / 100 \mathrm{~g}$ d'extrait $\mathrm{sec}$ ) des fromages ont été mesurées à l'aide d'un chloruremètre CORNING 926 à électrode soluble d'argent étalonné par rapport à la Norme FIL, 17A/1972.

\section{c) Résultats}

Le tableau 4 résume les caractéristiques moyennes des différents affinages. Il faut souligner la présence d'un affinage long à basse température (A1) proche des températures d'affinage du Beaufort. On constate une variabilité assez grande de la composition moyenne en $\mathrm{CO}_{2}$ et $\mathrm{NH}_{3}$ qui varient respectivement de 0,1 à $1,7 \%$ et de 35 à $62 \mathrm{ppm}$ pour les affinages $\mathrm{A} 1$ et $\mathrm{A} 3$.

\section{TABLEAU IV}

Moyenne et écart-type des paramètres physiques et chimiques caractérisant les quatre types d'affinage

Mean and standard deviation of the physical and chemical parameters characterizing the four cheese ripening operations

\begin{tabular}{l}
\hline \multirow{2}{*}{$\begin{array}{c}\text { Caractéristiques } \\
\text { Characteristics }\end{array}$} \\
\cline { 3 - 6 }
\end{tabular}

On note également de grandes différences dans l'intensité du salage en cave d'affinage, avec des rapports de $\mathrm{NaCl}$ variant de $0,17 \%$ (A2) à $0,54 \%$ (A4). 
Bien que les courbes ne soient pas présentées ici, on peut souligner une assez graide stabilité des conditions d'affinage propres à chaque cave au cours de la période expérimentale, avec toutefois une variabilité parfois élevée (A3) dans les apports ae sel.

Reçu le 9 juillet 1986. Accepté pour publication le 7 novembre 1986.

\section{Références bibliographiques}

Bachacou J., Masson J.P., Millier C., 1981. Manuel de la programmation statistique AMANCE 81. Editions du Département de Biométrie C.N.R.F.-I.N.R.A., Champenoux.

MICROSTAT ITCF, 1984. Service des études statistiques et informatiques. Station expérimentale ITCF, Boigneville, 91720 Maisse.

Mocquot G., 1979. Review of the progress of Dairy Science : Swiss-type cheese. J. Dairy Res., $46,133-160$.

Pearce K.N., 1977. The complexometric determination of calcium in dairy products. N.Z. J. Dairy Sci. Technol., 12, 113-115.

Prton C., 1983. Origine des Pseudomonas psychrotrophes du lait cru et conditions de leur multiplication à basse température au cours de la conservation du lait à la ferme. Mémoire de DEA, Faculté de Pharmacie de Châtenay-Malabry.

Richardson B.C., Pearce K.N., 1981. The determination of plasmin in dairy products. N.Z.J. Dairy Sci. Technol., 16, 209-220.

Rowland S.J., 1938. The determination of nitrogen distribution in milk. J. Dairy Res., 9, 42-46.

Wildbrett G., Kiermeier F., 1960. Distribution de la matière sèche et de la graisse dans les grandes meules de fromages. Z. Lebensm. Unters Forsch., 111, 6-20. 